\title{
Roseburia intestinalis-derived flagellin ameliorates colitis by targeting miR-223-3p-mediated activation of NLRP3 inflammasome and pyroptosis
}

\author{
XING WU ${ }^{1,2}$, SHIYU PAN ${ }^{1,2}$, WEIWEI LUO ${ }^{1,2}$, ZHAOHUA SHEN $^{1,2}$, XIANGRUI MENG $^{1,2}$, \\ MENGWEI XIAO $^{1,2}$, BEI TAN ${ }^{1,2}$, KAI NIE $^{1,2}$, TING TONG $^{1,2}$ and XIAOYAN WANG ${ }^{1,2}$ \\ ${ }^{1}$ Department of Gastroenterology, The Third Xiangya Hospital of Central South University, Changsha, Hunan 410013; \\ ${ }^{2}$ Hunan Key Laboratory of Non-Resolving Inflammation and Cancer, Changsha, Hunan 410008, P.R. China
}

Received October 28, 2019; Accepted June 4, 2020

DOI: $10.3892 / \mathrm{mmr} .2020 .11351$

\begin{abstract}
Ulcerative colitis (UC) is a type of inflammatory bowel disease (IBD), which is a chronic, relapsing condition associated with the disorder of gut microbial communities. A previous study reported that levels of Roseburia intestinalis (R.I), a butyrate-producing bacterium, are significantly decreased in patients with IBD and exert an anti-inflammatory function in dextran sulfate sodium (DSS)-induced colitis. However, the role of R.I flagellin in UC and its underlying molecular mechanism are not yet fully understood. Therefore, a DSS-induced colitis model in C57Bl/6 mice and the LPS/ATP-induced THP-1 macrophages were treated with $R . I$ flagellin, which were used to investigate the anti-inflammatory effects of R.I flagellin. The results demonstrated that R.I flagellin decreased colitis-associated disease activity index, colonic shortening and the pathological damage of the colon tissues in murine colitis models. Furthermore, R.I flagellin decreased the serum levels of proinflammatory cytokines and inhibited activation of the nucleotide-binding oligomerization segment-like receptor family 3 (NLRP3) inflammasome in murine colitis. $R . I$ flagellin was also demonstrated to decrease the Gasdermin D to yield the N-terminal fragment membrane pore and inhibit inflammasome-triggered pyroptosis. In vitro analysis indicated that microRNA (miR)-223-3p was involved in the regulation of $R . I$ flagellin on NLRP3 inflammasome activation. Taken together, the results of the present study demonstrated that R.I flagellin inhibited activation of the NLRP3 inflammasome and pyroptosis via miR-223-3p/NLRP3 signaling in macrophages, suggesting that $R . I$ flagellin may be used as a novel probiotic product for the treatment of UC.
\end{abstract}

Correspondence to: Professor Xiaoyan Wang, Department of Gastroenterology, The Third Xiangya Hospital of Central South University, 138 Tongzipo Road, Changsha, Hunan 410013, P.R. China E-mail: wangxiaoyan2913@126.com

Key words: ulcerative colitis, Roseburia intestinalis, flagellin, NLRP3, pyroptosis

\section{Introduction}

Ulcerative colitis (UC) is a chronic and recurrent autoimmune disease, which continues to exert multidimensional burdens on patients and health care systems (1). Pathogenesis of UC is associated with intestinal microbiota, immune response dysregulation, gene variants and environmental risk factors, such as smoking (2). Despite advancements in the treatment of this chronic condition, these clinical symptoms frequently reoccur, along with persistent side effects, such as opportunistic infections (3). Thus, novel effective therapeutic strategies, such as biological therapy, are required to improve the prognosis of patients with UC.

Intestinal microbiota play a key role in the pathogenesis of UC $(4,5)$. A previous study reported that the levels of Roseburia intestinalis (R.I) significantly decrease in patients with IBD, and alleviate colitis pathology by maintaining the Treg/Th17 balance and intestinal epithelial barrier in murine colitis models (6-8). Flagellins are important structural components of bacterial flagellum and are reported to have beneficial effects on the intestinal epithelial barrier $(9,10)$. However, the role of $R . I$ flagellin in UC and its underlying molecular mechanism remain unknown.

Pyroptosis is a novel type of proinflammatory programmed cell death, which differs from necrosis and apoptosis, and is closely associated with activation of the nucleotide-binding oligomerization segment-like receptor family 3 (NLRP3) inflammasome $(11,12)$. Following stimulation, NLRP3 combines with the adaptor protein, apoptosis-associated speck-like protein (ASC), which recruits caspase-1 to form the inflammasome complex (13-15). Subsequently, proximity-induced self-cleavage converts caspase- 1 into cleaved caspase-1, which in turn cleaves pro-interleukin (IL)-1 $\beta$, an inactive precursor of pro-inflammatory cytokine IL-1 $\beta$, into biologically active IL-1 $\beta$ (16). Cleaved caspase-1 also cleaves Gasdermin D to yield the N-terminal fragment (GSDMD-N), which forms membrane pores to trigger the release of IL-1 $\beta(17,18)$. Increasing evidence suggests that activation of the NLRP3 inflammasome and pyroptosis are involved in the inflammatory process of colitis $(19,20)$. However, the role of $R . I$ flagellin on the activation of the NLRP3 inflammasome and pyroptosis in UC remain unknown. 
In the present study, the effects of R.I flagellin on dextran sulfate sodium (DSS)- induced colitis and inflammasome activation were investigated both in vivo and in vitro. In addition, the role of $R . I$ flagellin on inflammasome-triggered pyroptosis was analyzed via western blotting and Transmission electron microscopy (TEM). Moreover, a microRNA (miRNA/miR) inhibitor was used to study the role of miR-223-3p on the signaling mechanisms of $R$.I flagellin. The present findings suggested that $R . I$ flagellin may be used as a novel probiotic product for the treatment of UC.

\section{Materials and methods}

Mice. Male C57BL/6J mice (age, 6 weeks; weight, 17-18 g; $\mathrm{n}=18$ ) were purchased from the Animal Center at Xiangya School of Medicine (Changsha, China) and housed in specific pathogen-free conditions at $22-26^{\circ} \mathrm{C}$, under a $12 / 12 \mathrm{~h}$ light/dark cycle with $40-70 \%$ humidity and ad libitum access to food and water. Animal health and behavior were monitored daily. The following humane endpoints were established for euthanasia: i) The inability to access food or water for $\sim 24 \mathrm{~h}$, ii) the inability to remain upright for $\sim 24 \mathrm{~h}$ and iii) a $20 \%$ decrease in optimal body weight. All experimental protocols were approved by the Ethics Committee of Medical Research, Third Xiangya Hospital of Central South University (approval no. 2018-S092), and were performed in accordance with the National Institutes of Health Guidelines for the Care and Use of Laboratory Animals.

Experimental design. Mice were randomly divided into three groups ( $\mathrm{n}=6 /$ group) as follows: Control group, DSS group and $\mathrm{DSS}+R . I$ flagellin group. Acute colitis was induced by administrating 3\% (w/v) DSS (molecular weight, 36,000-50,000; MP Biomedicals, Inc.) in drinking water for 7 days. Based on a previous study, $R$.I flagellin was administered once a day via intraperitoneal injection, at a dose of $50 \mathrm{mg} / \mathrm{kg}$ in $150 \mu \mathrm{l}$ normal saline to the wild-type (WT) mice with DSS-induced colitis from day 0 to 7 (21). The control and DSS groups received $150 \mu \mathrm{l}$ normal saline. On day $7, \sim 0.4 \mathrm{ml}$ of peripheral blood was collected from the eyes of the mice, under anesthesia. Subsequently, all mice were sacrificed by cervical dislocation and mortality was confirmed by cardiac arrest, respiratory arrest and complete loss of nerve reflex. Then, the peritoneum was opened and colon and spleen tissues were collected. Colon tissues were quickly used for western blotting or reverse transcription-quantitative (RT-q)PCR. In addition, colon tissues were fixed in $4 \%$ phosphate-buffered formaldehyde solution at $4^{\circ} \mathrm{C}$ for $24 \mathrm{~h}$, embedded in paraffin and then used for histopathological analysis and immunofluorescence.

Disease activity index (DAI) and histological scorings of colitis. Mice were weighed daily and visually inspected for diarrhea and rectal bleeding to assess the DAI. The DAI score was calculated as the mean of three parameters: Weight loss, 0-4 (0, no loss; 1, 1-5\% loss; 2, 6-10\% loss; 3, 11-20\% loss; 4, >20\% weight loss); stool consistency, 0-4 (0, normal; 2 , loose stool; 4, diarrhea) and stool bleeding, 0-4 (0, no blood; 2, presence; 4, gross blood). For hematoxylin and eosin detection, colon tissue samples were fixed in PBS containing $10 \%$ neutral-buffered formalin (Shanghai Rongbai biological technology Co., Ltd.) at $4^{\circ} \mathrm{C}$ for $24 \mathrm{~h}$, embedded in paraffin, cut into $5-\mu \mathrm{m}$ thick sections and stained with hematoxylin for $30 \mathrm{sec}$ and eosin for $2 \mathrm{~min}$ (both at room temperature). A light microscope (magnification, x100; DP72; Olympus Corporation) was used to observe the colonic histopathology. Histopathological changes (the changes of colonic mucosal ulceration, glandular defects and lymphocyte infiltration) were evaluated using a previously established scoring system (22).

Immunofluorescence. Colonic tissue sections were incubated with rabbit anti-NLRP3 primary antibody (1:200; Servicebio, Inc.; cat. no. GB11300) overnight at $4^{\circ} \mathrm{C}$. Sections were subsequently washed with $0.01 \mathrm{M}$ PBS three times, prior to incubation with $\mathrm{Cy} 3$ conjugated donkey anti-rabbit secondary antibody (1:200; Servicebio, Inc.; cat. no. GB21403; http://www.servicebio.cn/goodsdetail?id=255) for $1 \mathrm{~h}$ at room temperature. Sections were re-washed with $0.01 \mathrm{M} \mathrm{PBS}$, stained with $2.5 \mu \mathrm{g} / \mathrm{ml}$ DAPI for $20 \mathrm{~min}$ at room temperature, and observed under a confocal laser scanning microscope (Olympus Corporation) at x100 magnification.

Cell culture and reagents. THP-1 cells were purchased from the American Type Culture Collection and maintained in RPMI-1640 medium (Gibco; Thermo Fisher Scientific, Inc.) supplemented with $10 \%$ FBS (Gibco; Thermo Fisher Scientific, Inc.), at $37^{\circ} \mathrm{C}$ in a humidified incubator of $5 \% \mathrm{CO}_{2}$. Cells were differentiated into macrophages via incubation with $150 \mathrm{ng} / \mathrm{ml}$ phosphomolybdic acid (Sigma-Aldrich; Merck KGaA) for $36 \mathrm{~h}$ at $37^{\circ} \mathrm{C}$. The following concentrations of agonists or stimuli were used: $1 \mu \mathrm{g} / \mathrm{ml}$ LPS (Sigma-Aldrich; Merck KGaA), $5 \mathrm{mM}$ ATP (Sigma-Aldrich; Merck KGaA) and 200 ng/ml flagellin (Detai Biologics Co., Ltd.).

$R T-q P C R$. Total RNA from colon, peritoneal macrophages or THP-1 cells was extracted using TRIzol reagent (Invitrogen; Thermo Fisher Scientific, Inc.), according to the manufacturer's protocol and reverse-transcribed into cDNA using the Reverse Transcription kit (Thermo Fisher Scientific, Inc.). The temperature protocol for miRNA reverse transcription was: $42^{\circ} \mathrm{C}$ for $60 \mathrm{~min}$, followed by $70^{\circ} \mathrm{C}$ for $5 \mathrm{~min}$. qPCR was subsequently performed using the SYBR Green qPCR Master mix (Vazyme Biotech Co., Ltd.). miRNA expression levels were assessed using the Mir-X miRNA First-Strand Synthesis and Mir-X miRNA qRT-PCR TB Green kits (Takara Biotechnology Co., Ltd.). The temperature protocol for miRNA reverse transcription was: $37^{\circ} \mathrm{C}$ for $60 \mathrm{~min}$, followed by $85^{\circ} \mathrm{C}$ for $5 \mathrm{~min}$ and stored at $4^{\circ} \mathrm{C}$. The primer sequences used for qPCR are presented in Table SI. The following thermocycling conditions were used for qPCR: Initial denaturation: $95^{\circ} \mathrm{C}$ for $30 \mathrm{sec}$; followed by 40 cycles at $95^{\circ} \mathrm{C}$ for $5 \mathrm{sec}$ and $60^{\circ} \mathrm{C}$ for $30 \mathrm{sec}$; Dissociation Curve: $95^{\circ} \mathrm{C}$ for $60 \mathrm{sec}, 55^{\circ} \mathrm{C}$ for $30 \mathrm{sec}$ and $95^{\circ} \mathrm{C}$ for $60 \mathrm{sec}$. Relative gene expression levels were calculated using the $2^{-\Delta \Delta \mathrm{Cq}}$ method (23). mRNA expression was normalized to GAPDH, while miRNA expression was normalized to the small nuclear RNA, U6.

Prediction of miRNAs that can bind to NLRP3. The database of miRDB (http://www.mirdb.org; version, 5.0) and TargetScan (http://www.targetscan.org; version, 7.2) was used to identify miRNAs which can bind to 3'-untranslated region (UTR) 
of NLRP3. Predictions of these two databases identified miR-5197-3p, miR-223-3p, miR-589-3p and miR-1305 as top miRNA candidates. The sequences of miRNA were obtained from TargetScan.

Enzyme-linked immunosorbent assay (ELISA). The expression levels of IL-1 $\beta$, IL-18, TNF- $\alpha$ and IL- 6 in mouse serum were measured via ELISA (IL-1 $\beta$, cat. no. PMLB00C; IL-18, cat. no, 7625; TNF- $\alpha$, cat. no, PMTA00B; IL-6, cat. no, PM6000B; R\&D Systems, Inc.), according to the manufacturer's protocol. All experiments were performed twice and repeated three times.

Western blotting. Total protein was extracted from colonic tissue samples and THP-1 cells by homogenization in RIPA Lysis Buffer (cat. no. CW2333; CoWin Biosciences), as previously described (6). Protein concentration was determined using a bicinchoninic acid assay (Thermo Fisher Scientific, Inc.). Equal amounts of proteins $(40 \mu \mathrm{g})$ were electrophoresed on $12 \%$ SDS-PAGE gels and subsequently transferred onto polyvinylidene difluoride membranes (Merck KGaA). Membranes were blocked with 5\% BSA (Wuhan Goodbio Technology Co., Ltd.) at room temperature for $1 \mathrm{~h}$, and then incubated with primary antibodies against: NLRP3 (cat. no. ab214185; Abcam), ASC (cat. no. 13833; Cell Signaling Technology, Inc.), cleaved caspase-1 (cat. no. 4199; Cell Signaling Technology, Inc.), GSDMD-N (cat. no. 93709; Cell Signaling Technology, Inc.) and GAPDH (cat. no. 10494-1-AP; ProteinTech Group, Inc.) overnight at $4^{\circ} \mathrm{C}$ (all 1:1,000). Following the primary incubation, membranes were incubated with HRP-conjugated Affinipure Goat Anti-Rabbit/Mouse IgG secondary antibody for $1 \mathrm{~h}$ at room temperature (1:5,000; cat. nos. SA00001-2 and SA00001-2; ProteinTech Group, Inc.). Protein bands were visualized using the ECL kit (Merck KGaA). Densitometry data were analyzed using ImageJ (version $1.52 \mathrm{~V}$; National Institutes of Health).

TEM. THP-1 cells were fixed with $2.5 \%$ glutaraldehyde in $4^{\circ} \mathrm{C}$ for $24 \mathrm{~h}$, washed with PBS three times and subsequently fixed with $1 \%$ osmium tetroxide in $4^{\circ} \mathrm{C}$ for $2 \mathrm{~h}$. Following dehydration in a descending ethanol series (30, 50, 70, 90 and 100\%), samples were embedded in Epon/Araldite mixture and subsequently cut into 50-60-nm-thick sections. Sections were stained with uranium acetate and lead citrate (these were performed by the TEM assistants at the Central South University), and observed under a transmission electron microscope at x20,000 magnification (cat. no. HT7700; Hitachi, Ltd.).

Transfection. hsa-miR-223-3p mimic, hsa-miR-223-3p inhibitor, Toll-like receptor 5 (TLR5) small interfering (si)RNA and scrambled negative control (NC) were synthesized by Shanghai GenePharma Co., Ltd. The sequences are presented in Table SI. Transfection was performed using Lipofectamine 3000 reagent (Invitrogen; Thermo Fisher Scientific, Inc.). The concentration of miRNA mimics, inhibitor, NC and si-TLR5 in these experiment was $100 \mathrm{nM}$. THP-1 cells were collected for further experimentation, $48 \mathrm{~h}$ post-transfection.

Dual-luciferase reporter assay. The reporter plasmids, pmirGLO-NLRP3-3'-UTR, pmirGLO-NLRP3-3'-UTR-MUT and pmirGLO-NC were purchased from Shanghai GenePharma Co., Ltd. THP-1 cells were co-transfected with the plasmids and hsa-miR-223-3p mimic/inhibitor using Lipofectamine 3000 reagent (Invitrogen; Thermo Fisher Scientific, Inc.). Following incubation at $37^{\circ} \mathrm{C}$ for $24 \mathrm{~h}$, luciferase activities were detected using a Dual-Luciferase Reporter assay system (Promega Corporation), and results were compared with Renilla luciferase activity.

Statistical analysis. Statistical analysis was performed using GraphPad Prism software (version 7.0; GraphPad Software Inc.). Each experiment was repeated three times. Data are presented as the mean \pm standard error of the mean. One-way ANOVA, followed by least significant difference post-hoc test were performed to compare differences between multiple groups. $\mathrm{P}<0.05$ was considered to indicate a statistically significant difference.

\section{Results}

R.I flagellin attenuates inflammation in a DSS-induced colitis model. In order to investigate the therapeutic effects of $R . I$ flagellin in vivo, a DSS-induced acute colitis mouse model was established. DSS administration to 6-week-old mice induced severe colitis, which was characterized by increased loss of body weight and higher DAI score compared with the healthy control mice. Notably, administration of R.I flagellin significantly restored body weight (Fig. 1A) and DAI score (Fig. 1B) in DSS-treated mice. Furthermore, the vital indicators of colitis, colonic shortening (Fig. 1C and D) and splenomegaly (Fig. 1E), were evidently mitigated following administration of $R . I$ flagellin. Consistent with the remission of clinical signs, treatment with $R$.I flagellin decreased mucosal ulceration, glandular defects and lymphocyte infiltration in colonic tissues (Fig. 1F and G). Furthermore, treatment with R.I flagellin significantly inhibited DSS-induced increased serum levels of IL-1 $\beta$, IL-18, TNF- $\alpha$ and IL-6 (Fig. 2A-D). Collectively, these results indicated that $R$.I flagellin is protective against DSS-induced colitis.

\section{R.I flagellin decreases NLRP3 inflammasome activation} in DSS-induced mice. RT-qPCR analysis was performed to assess the expression levels of NLRP3, ASC and IL-1 $\beta$ in colonic tissues, to determine whether R.I flagellin relieves inflammation by regulating activation of the NLRP3 inflammasome. The results demonstrated that the mRNA expression levels of NLRP3, ASC and IL-1 $\beta$ were upregulated in the DSS group compared with the control group, which was significantly reversed following treatment with $R$.I flagellin (Fig. 3A-D). Similarly, immunofluorescence staining and western blot analysis indicated that NLRP3 distinctly increased in the DSS group, was significantly counteracted following administration of R.I flagellin (Fig. 3D and E). Furthermore, western blot analysis demonstrated that ASC protein expression significantly increased in the DSS group, was significantly reversed following treatment with R.I flagellin (Fig. 3F). ELISA indicated that treatment with $R . I$ flagellin significantly inhibited DSS-induced increased serum levels of IL-1 $\beta$ (Fig. $2 \mathrm{~A}$ ); it has been shown that IL-1 $\beta$ is associated with the NLRP3 inflammasome (24). 
A

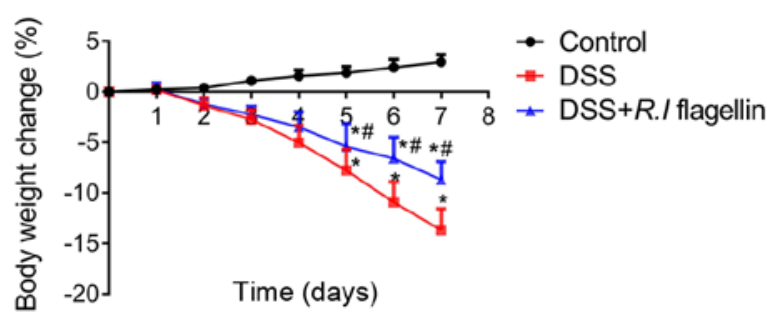

B

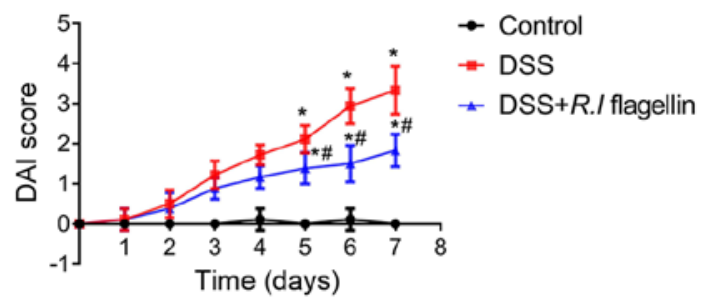

C

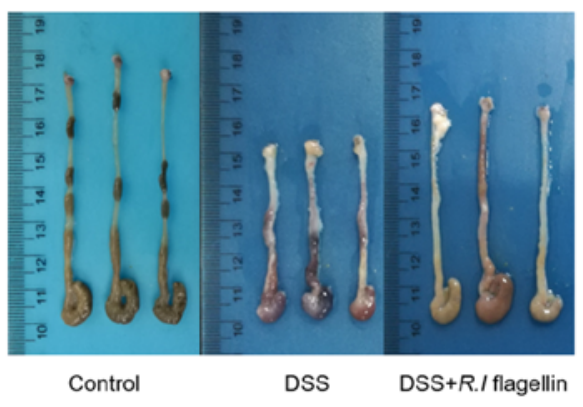

$\mathbf{F}$

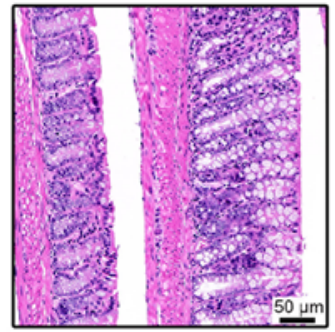

Control

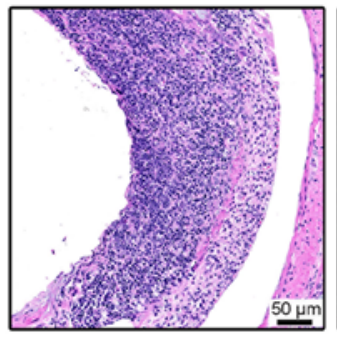

DSS
D
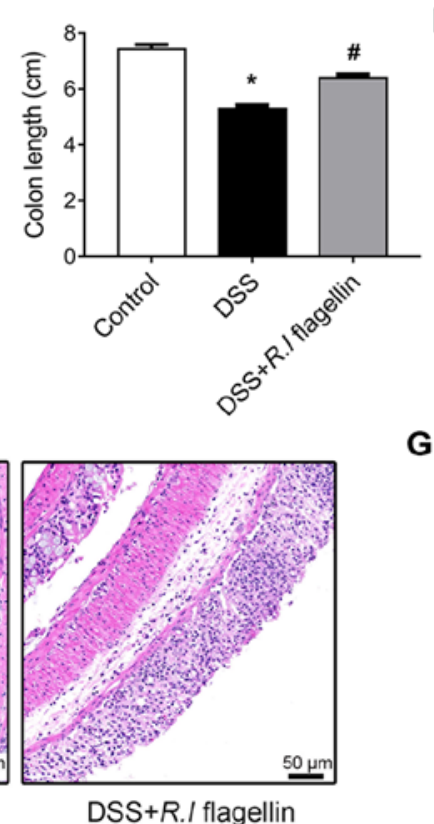

G
E
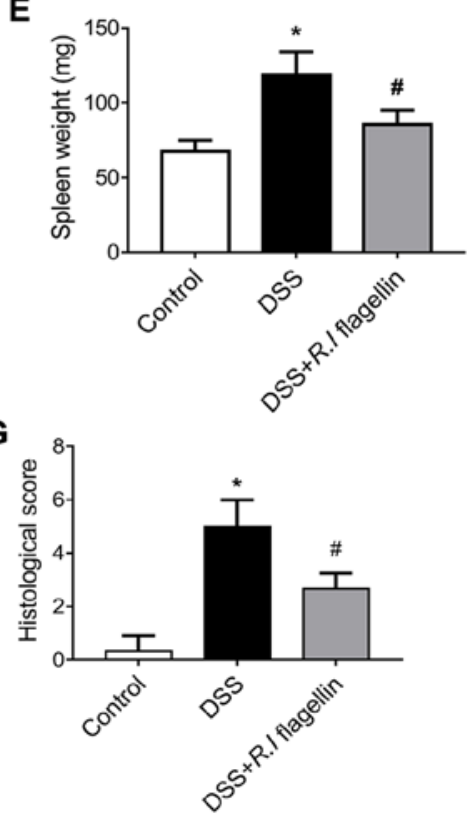

Figure 1. R.I flagellin ameliorates DSS-induced colitis. (A) Body weight changes with disease progression. (B) DAI score was assessed following treatment with DSS. (C and D) Representative images of colon length from each cohort. (E) Weight of spleen from each cohort. (F) Representative images of hematoxylin and eosin stained colon sections and $(\mathrm{G})$ histological analysis of colons from each cohort. Magnification, $\mathrm{x} 200$. Data are presented as the mean \pm standard error of the mean ( $\mathrm{n}=6 /$ group). ${ }^{*} \mathrm{P}<0.05$ vs. control group; ${ }^{*} \mathrm{P}<0.05$ vs. DSS group. R.I, Roseburia intestinalis; DSS, dextran sulfate sodium; DAI, disease activity index.
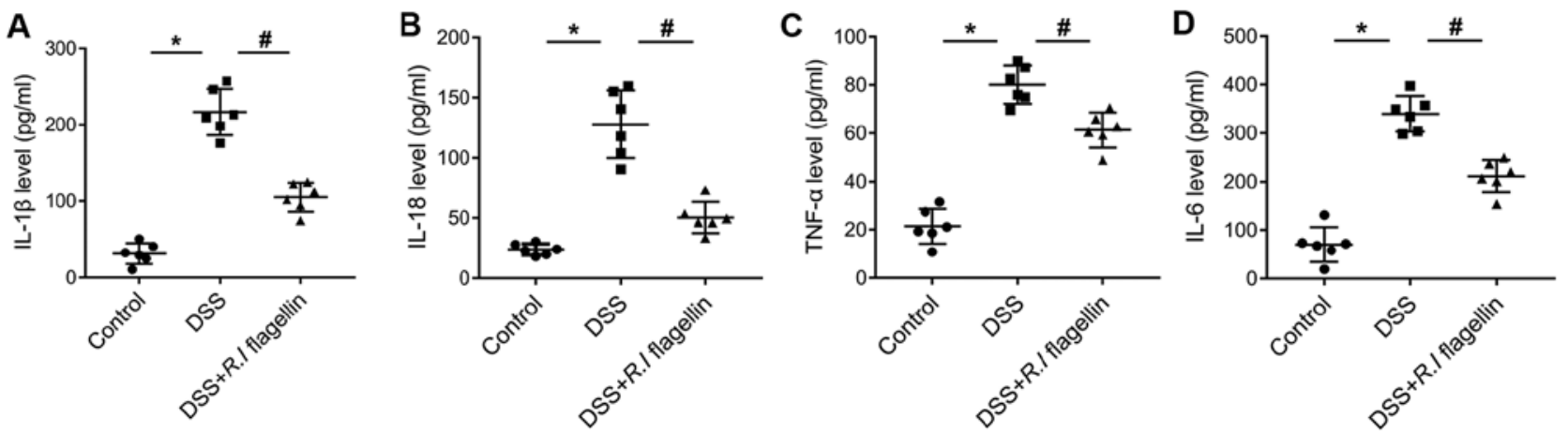

Figure 2. Regulation of $R$.I flagellin on proinflammatory cytokines. Serum expression levels of (A) IL-1 $\beta$, (B) IL-18, (C) TNF- $\alpha$ and (D) IL-6 were determined via the enzyme-linked immunosorbent assay. Each symbol represents a respective mouse. Data are presented as the mean \pm standard error of the mean ( $\mathrm{n}=6$ /group). ${ }^{*} \mathrm{P}<0.05$ vs. control group; ${ }^{~} \mathrm{P}<0.05$ vs. DSS group. R.I, Roseburia intestinalis; IL, interleukin; DSS, dextran sulfate sodium.

Collectively, these results indicated that $R . I$ flagellin affected the inflammasome activation of colon tissue.

R.I flagellin decreases NLRP3 inflammasome activation and pyroptosis in THP-1 macrophages. Macrophages are the gatekeepers of intestinal immune homeostasis $(25,26)$. The present study further investigated the regulation of $R$.I flagellin on inflammasome activation in THP-1 macrophages. Transcript and protein expression levels of NLRP3 and ASC significantly increased following treatment with LPS+ATP, the effects of which were significantly reversed following treatment with $R . I$ flagellin (Fig. 4A-E). Furthermore, IL-1 $\beta$ mRNA expression and secretion significantly increased following treatment with LPS+ATP compared with the control, the effects of which 

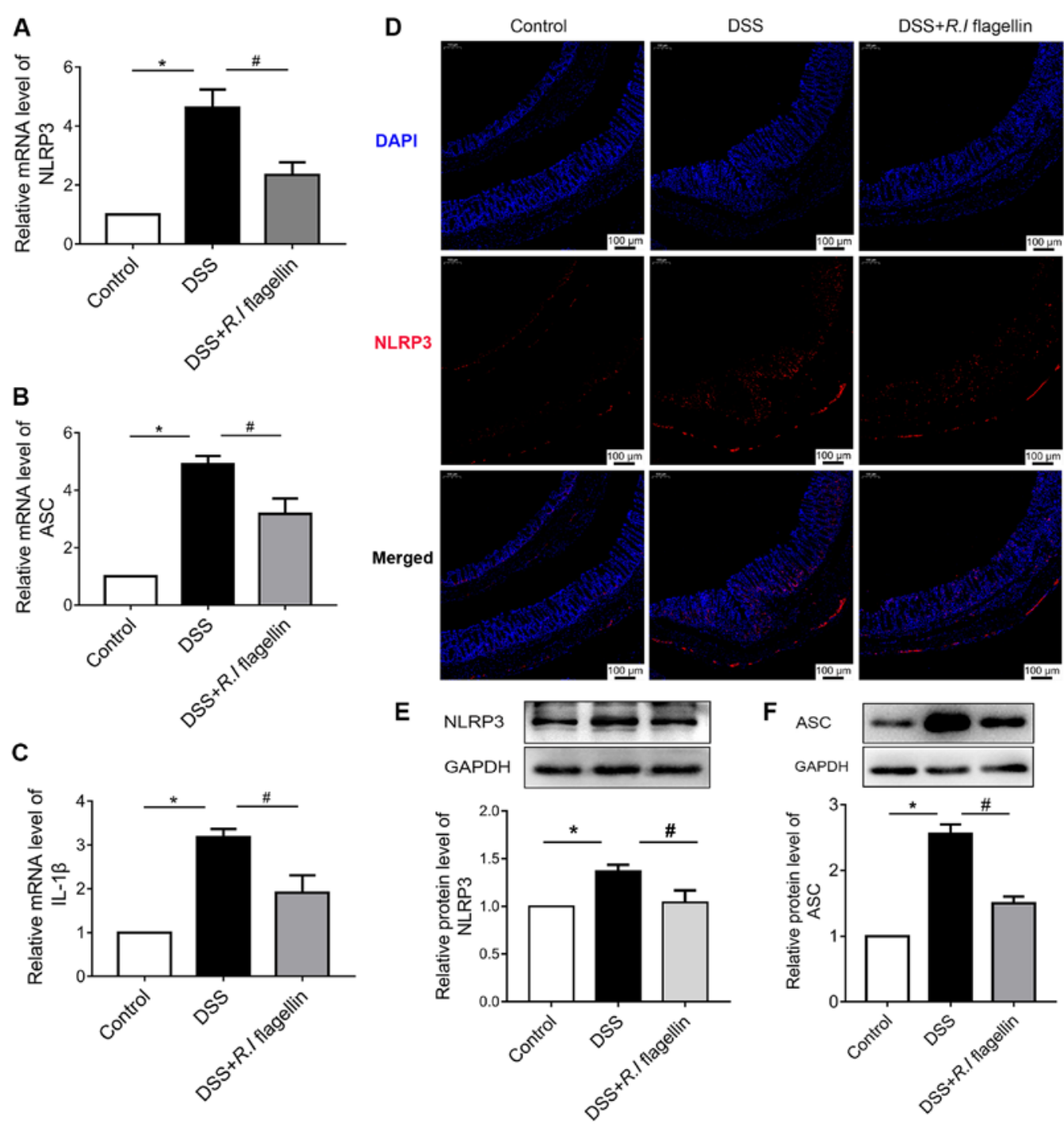

Figure 3. R.I flagellin inhibits NLRP3 inflammasome activation in colonic tissues. Reverse transcription-quantitative PCR analysis was performed to determine mRNA expression levels of (A) NLRP3, (B) ASC and (C) IL-1 $\beta$ in colonic tissues. (D) Paraffin-embedded colon sections were stained for NLRP3 (red) and nuclei were stained with DAPI (blue). Magnification, x100. Western blot analysis was performed to determine the protein expression levels of (E) NLRP3 and (F) ASC in colonic tissues. Data are presented as the mean \pm standard error of the mean ( $\mathrm{n}=3 /$ group). ${ }^{*} \mathrm{P}<0.05$ vs. control group; ${ }^{*} \mathrm{P}<0.05$ vs. DSS group. R.I, Roseburia intestinalis; NLRP3, nucleotide-binding oligomerization segment-like receptor family 3; ASC, apoptosis-associated speck-like protein; DSS, dextran sulfate sodium; IL, interleukin.

were significantly counteracted following administration of $R . I$ flagellin (Fig. 4H and I). Collectively, these results confirmed that $R . I$ flagellin can reverse the LPS+ATP- induced NLRP3 inflammasome activation in THP-1 macrophages.

The regulation of $R . I$ flagellin in inflammasome-triggered pyroptosis was also investigated. Expression of the vital markers of pyroptosis, cleaved caspase-1 and GSDMD-N, significantly increased following LPS+ATP treatment; however, the expression levels significantly decreased following treatment with R.I flagellin (Fig. 4C, F and G). TEM analysis confirmed that GSDMD-N membrane pore decreased following administration of $R$.I flagellin in LPS+ATP-induced pyroptosis (Fig. 4J). Collectively, these results indicated that $R . I$ flagellin alleviated LPS+ATP-induced NLRP3 inflammasome activation and pyroptosis.

Silencing miR-223-3p reverses the effect of $R$.I flagellin on NLRP3 inflammasome activation and pyroptosis. In order to determine the underlying molecular mechanism by which R.I flagellin mediates regulation of NLRP3 inflammasome activation in human macrophages, the present study identified microRNAs (miRNAs/miRs) that bind to NLRP3, using the miRDB (http://www.mirdb.org) and TargetScan (http://www. targetscan.org) databases. THP-1 cells were treated with LPS+ATP and/or R.I flagellin for $12 \mathrm{~h}$. As presented in Fig. 5A, treatment with LPS+ATP significantly downregulated the expression levels of miR-223-3p and miR-589-3p compared with the control group. However, only miR-223-3p exhibited a significant increase in expression following treatment with R.I flagellin, compared with the LPS+ATP group. The other miRNAs (miR-5197-3p and miR-1305) were not markedly altered.

The binding sequence of miR-223-3p in the NLRP3 3'-UTR was predicted to determine if NLRP3 was a downstream target of miR-223-3p in THP-1 cells (Fig. 5B). As presented in Fig. 5C, the dual-luciferase reporter assay demonstrated that binding of 
A

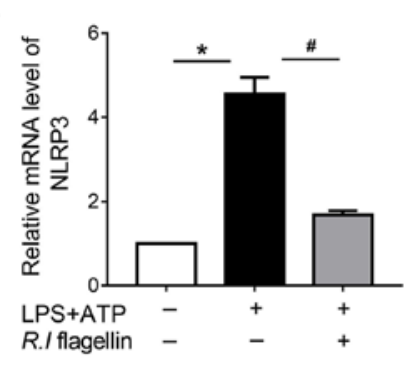

D

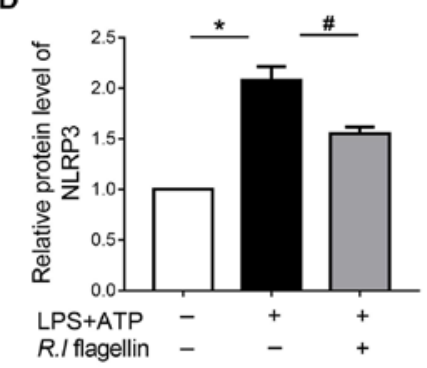

G

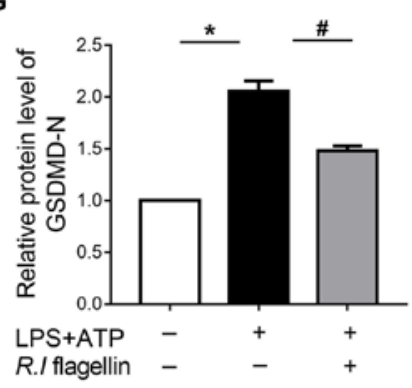

B

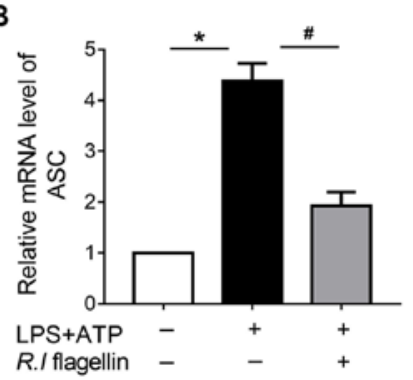

E

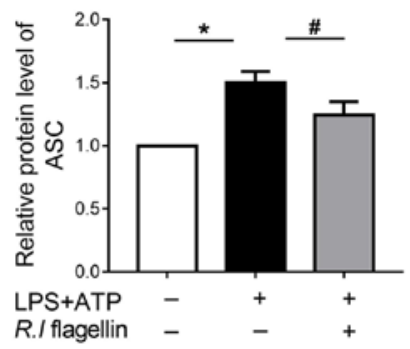

H

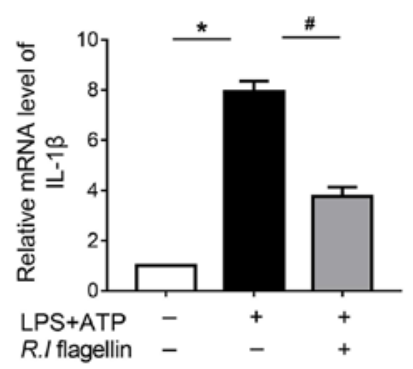

C LPS+ATP $\quad-\quad+\quad+$

R./ flagellin $\quad-\quad-\quad+$

NLRP3

ASC

Cleaved

caspase-1

GSDMD-N

GAPDH

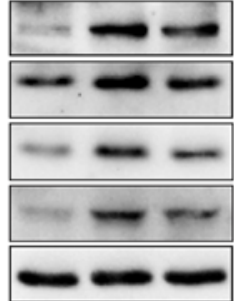

F

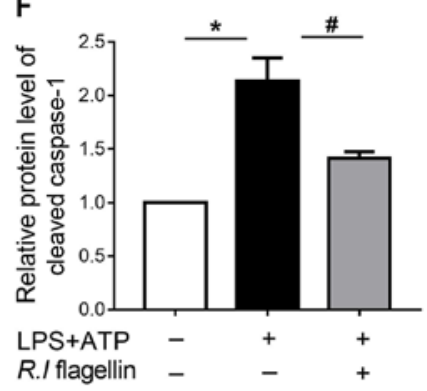

I

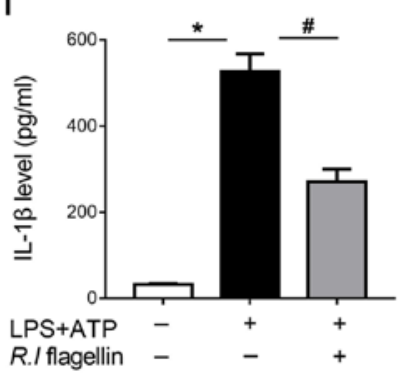

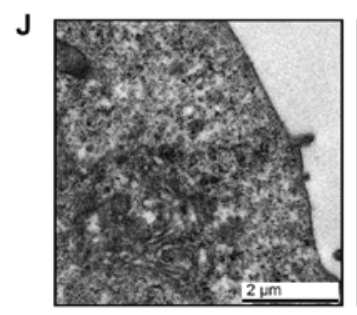

Control

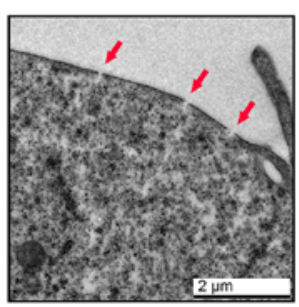

LPS+ATP

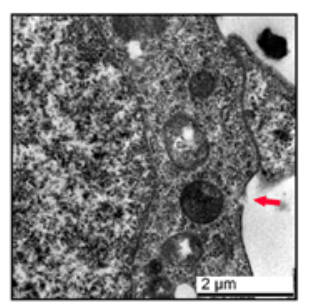

LPS + ATP $+R$.I flagellin

Figure 4. R.I flagellin decreases NLRP3 inflammasome activation and pyroptosis in THP-1 macrophages. RT-qPCR analysis was performed to determine mRNA expression levels of (A) NLRP3 and (B) ASC. (C) Western blot analysis was performed to determine the protein expression levels of (D) NLRP3, (E) ASC, (F) cleaved caspase-1 and (G) GSDMD-N in THP-1 macrophages. (H) RT-qPCR analysis was performed to determine IL-1 $\beta$ mRNA expression in THP-1 macrophages. (I) IL-1 $\beta$ levels in the culture medium were detected via the enzyme-linked immunosorbent assay. (J) Representative electron micrographs of treated THP-1 macrophages (magnification, $\mathrm{x} 20,000$ ). Red arrowheads indicate membrane pores. Data are presented as the mean \pm standard error

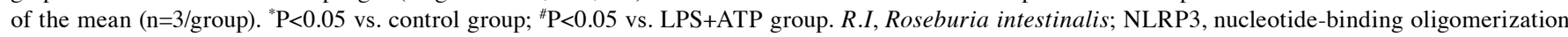
segment-like receptor family 3; RT-qPCR, reverse transcription-quantitative PCR; ASC, apoptosis-associated speck-like protein; GSDMD-N, Gasdermin D N-terminal fragment; IL, interleukin.

miR-223-3p mimic to the WT NLRP3 3'-UTR significantly decreased luciferase activity, while binding of miR-223-3p inhibitor to the WT NLRP3 3'-UTR significantly increased luciferase activity in THP-1 cells, compared with the respective controls. However, binding of miR-223-3p mimic or inhibitor to MUT NLRP3 3'-UTR had no significant effect on luciferase activity.

In order to determine whether miR-223-3p was involved in activation of the NLRP3 inflammasome, THP-1 cells were treated with LPS+ATP and/or R.I flagellin for $12 \mathrm{~h}$ following transfection with miR-223-3p inhibitor for $24 \mathrm{~h}$. As anticipated, transfection with miR-223-3p inhibitor significantly downregulated miR-223-3p expression in THP-1 cells (Fig. 5D).
The inhibitory effect of $R$.I flagellin on NLRP3 was reversed by downregulating miR-223-3p (Fig. 5E-G). Furthermore, inhibiting miR-223-3p expression counteracted the restraint of R.I flagellin on the release of IL-1 $\beta$ (Fig. $5 \mathrm{H}$ ). Collectively, these results indicated that miR-223-3p was involved in the regulation of $R$.I flagellin on NLRP3 inflammasome activation in THP-1 cells.

The present study also investigated the effect of miR-223-3p on R.I flagellin-regulated pyroptosis. The results indicated that miR-223-3p knockdown reversed $R$.I flagellin-mediated downregulation of cleaved caspase- 1 and GSDMD-N (Fig. 5I-K). Collectively, these results indicated 
A

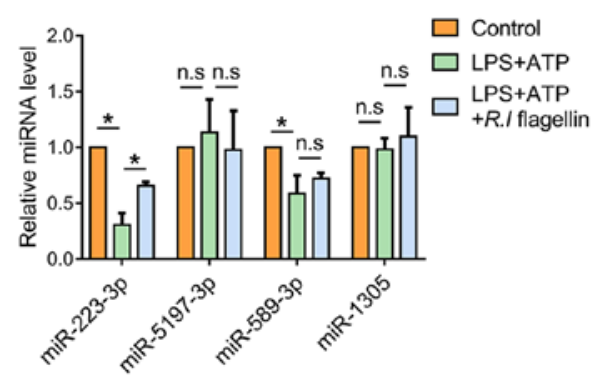

D

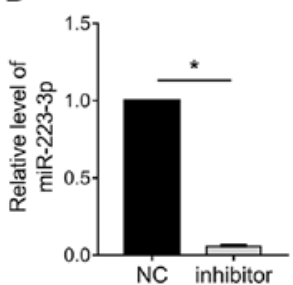

E

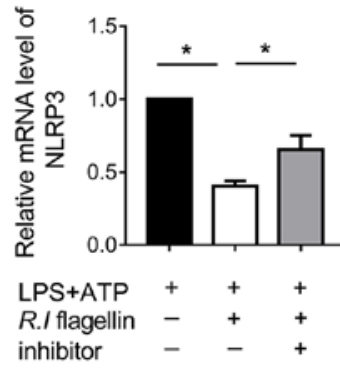

H

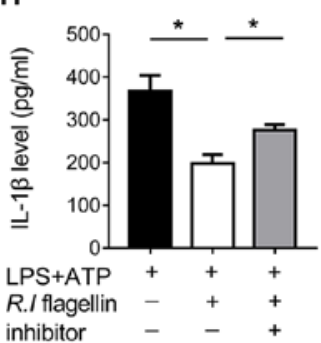

I

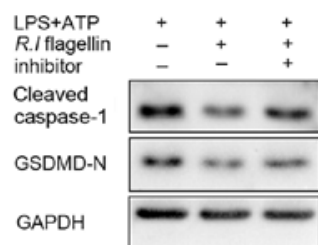

B

3' ACCCCATAAACTGT-TTGACTGT miR-223-3p

NLRP3 wide-type

5' ...CGCTATCTTTCTATTTTGACTGC... 3' UTR of NLRP3 mutant-type

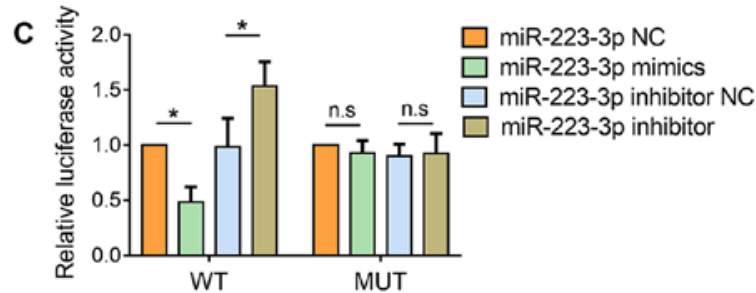

$\mathbf{F}$

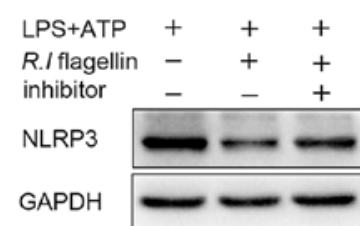

G

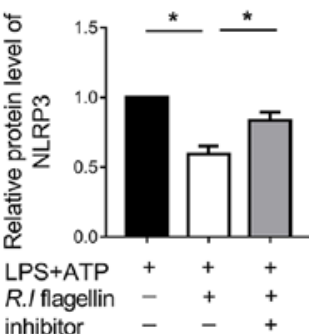

$\mathbf{J}$

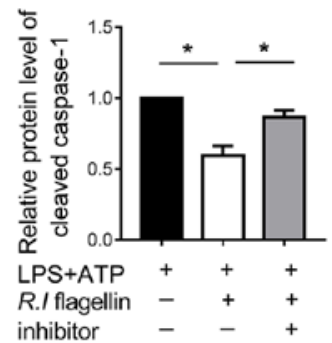

K

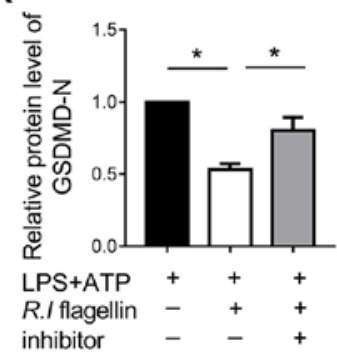

Figure 5. Silencing miR-223-3p reverses the effect of R.I flagellin on NLRP3 inflammasome activation and pyroptosis in THP-1 macrophages. (A) RT-qPCR analysis was performed to determine the expression levels of miR-223-3p, miR-5197-3p, miR-589-3p and miR-1305. (B) Schematic diagram of the predicted binding sequence of miR-223-3p in the 3'-UTR of NLRP3. (C) Luciferase activity for the WT and MUT 3'-UTR of NLRP3 pGL3 vector co-transfected with miR-223-3p NC, miR-223-3p mimics, miR-223-3p inhibitor NC and miR-223-3p inhibitor. RT-qPCR analysis was performed to determine the expression levels of (D) miR-223-3p and (E) NLRP3. (F and G) Western blot analysis was performed to determine NLRP3 protein expression. (H) IL-1 $\beta$ levels in the culture medium were detected via the enzyme-linked immunosorbent assay. (I) Western blot analysis was performed to determine the protein expression levels of (J) cleaved caspase-1 and (K) GSDMD-N. Data are presented as the mean \pm standard error of the mean ( $\mathrm{n}=3 / \mathrm{group})$. ${ }^{\mathrm{P}}<0.05$. miR/miRNA, microRNA; R.I, Roseburia intestinalis; NLRP3, nucleotide-binding oligomerization segment-like receptor family 3; RT-qPCR, reverse transcription-quantitative PCR; IL, interleukin; GSDMD-N, Gasdermin D N-terminal fragment; n.s, no significance; UTR, untranslated region; NC, negative control; WT, wild-type; MUT, mutant.

A

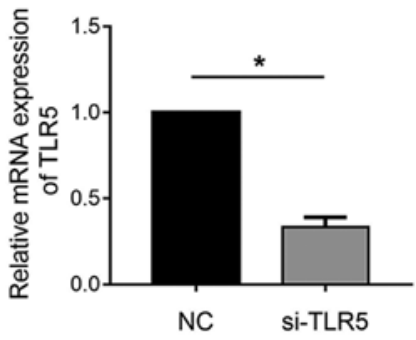

B

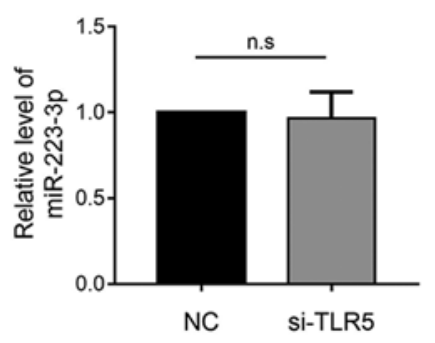

Figure 6. R.I flagellin may regulate miR-223-3p expression through TLRs but not TLR5. (A) RT-qPCR analysis was performed to determine mRNA TLR5 expression in THP-1 macrophages. (B) THP-1 macrophages were treated with LPS/ATP+R.I flagellin and transfected with si-TLR5. RT-qPCR analysis was performed to determine miR-223-3p expression. Data are presented as the mean \pm standard error of the mean ( $\mathrm{n}=3 / \mathrm{group})$. ${ }^{*} \mathrm{P}<0.05$. R.I, Roseburia intestinalis; miR, microRNA; TLR, Toll-like receptor; si, small interfering; NC, negative control; n.s, no significance.

that $R . I$ flagellin alleviated inflammasome-induced pyroptosis by inhibiting miR-223-3p/NLRP3 signaling in macrophages.

R.I flagellin may regulate miR-223-3p expression through TLRs but not TLR5. TLR5 acts as a pattern recognition receptor and responds to flagellin in order to modulate miRNA expression $(27,28)$. The present study aimed to investigate whether the regulation of miR-223-3p expression by $R$.I flagellin is mediated through the activity of TLR5. TLR5 gene expression was knocked down in THP-1 macrophages (Fig. 6A). However, 
transfection with siRNA-TLR5 had no effect on the regulation of miR-223-3p expression by R.I flagellin (Fig. 6B). Collectively, these results indicated that $R . I$ flagellin may regulate miR-223-3p expression through TLRs but not TLR5 specifically.

\section{Discussion}

The present study demonstrated that: i) R.I flagellin exhibited beneficial effects on colitis, ii) $R$.I flagellin inhibited inflammasome activation, iii) $R$.I flagellin was a negative regulator of inflammasome-triggered pyroptosis and (4) miR-223-3p was an upstream signal molecule of NLRP3 that plays a key role in the anti-inflammatory mechanism of $R$.I flagellin. Collectively, these results indicate a potential clinical application of R.I flagellin to relieve intestinal inflammation.

The disorder of gut microbial communities has been identified as the core defect that causes chronic intestinal inflammation $(4,5)$. Several studies have suggested that some of the intestinal bacteria play important roles in relieving intestinal inflammation and maintaining intestinal barrier integrity (29-31). A previous study demonstrated that R.I levels were significantly decreased in untreated patients with IBD. Furthermore, R.I was reported to alleviate colitis pathology by maintaining the Treg/Th17 balance in an experimental colitis model (6). However, the role of R.I flagellin, as the main structural component of R.I, in relieving colitis remains unclear. The results of the present study demonstrated that $R . I$ flagellin alleviated DSS-induced inflammation in a mouse model of colitis. Furthermore, cytokines such as IL-1 1 , IL-18, TNF- $\alpha$ and IL- 6 are significantly elevated in UC, some of which have become important therapeutic targets $(32,33)$. The results of the present study indicated that treatment with $R . I$ flagellin significantly inhibited these inflammatory cytokines, indicating that $R . I$ flagellin has a therapeutic effect on colitis. These results are consistent with the anti-inflammatory effect of R. intestinalis, suggesting that R.I-derived flagellin may be the key component of R.I in inhibiting inflammation.

Contrary to studies that suggest that flagellin may be an exogenous substance that triggers inflammation (34-36), increasing evidence indicates that flagellins play a protective role in inflammation and intestinal barrier functions $(9,10,37,38)$. For example, Salmonella typhimurium-derived flagellin can induce the secretion of anti-inflammatory factors, such as IL-22, from dendritic cells to protect intestinal epithelial barrier (9). Thus, flagellins from different sources may play different roles in regulating inflammatory immunity. The results of the present study confirm the role of flagellin as a biological agent for the treatment of colitis.

Recent studies suggest that pyroptosis plays a pivotal role in the occurrence and development of UC. Davis et al (39) demonstrated that intestinal epithelial cell pyroptosis is vital for the development of epithelial barrier dysfunction and mucosal inflammation. Furthermore, inhibition of pyroptosis may be a therapeutic mechanism of action for mesalamine and corticosteroids (19). However, little is known about the effect of probiotics and their components on pyroptosis. The results of the present study verified that $R . I$ flagellin can alleviate NLRP3 inflammasome activation and inflammasome-triggered pyroptosis.

miRNA-mediated regulation of several immune processes, including pyroptosis have been extensively studied and well established $(40,41)$. The present study used miRDB and TargetScan databases to identify miRNAs that have binding sites to NLRP3, and demonstrated that the decreased miR-223-3p expression was significantly altered following administration of $R . I$ flagellin. Furthermore, inhibiting miR-223-3p expression reversed the effects of $R . I$ flagellin on suppressing activation of the NLRP3 inflammasome and downregulating expression of pyroptosis-associated proteins. Collectively, these results indicated that R.I flagellin alleviated inflammasome-induced pyroptosis by inhibiting miR-223-3p/NLRP3 signaling.

Several studies have reported that TLR signaling can modulate miRNA expression $(27,42)$. TLR5 plays an important role in innate immune responses to flagellin $(28,43)$. However, the results of the present study demonstrated that transfection with si-TLR5 had no effect on the regulation of miR-223-3p expression by R.I flagellin. Previous studies have reported that flagellin is recognized by other receptors, such as TLR2, NLRC4 and TLR11 $(34,44,45)$. Thus, it is suggested that $R . I$ flagellin may regulate miR-223-3p expression through TLRs but not TLR5. Prospective studies will focus on investigating the association between other recognition receptors and R.I flagellin through experiments such as pull-down and mass spectrometry. Moreover, although this study preliminarily investigated the inhibitory effect of flagellin on colitis and its mechanism of inflammation suppression, its practical role in patients with UC remains to be elucidated; this is an important direction of follow-up research, and is crucial for the clinical transformation application of $R$.I flagellin.

In conclusion, the results of the present study demonstrated that $R . I$ flagellin relieved intestinal inflammation by inhibiting inflammasome-induced pyroptosis through miR-223-3p/NLRP3 signaling. Thus, R.I flagellin exhibits a beneficial probiotic role in ameliorating UC.

\section{Acknowledgements}

Not applicable.

\section{Funding}

The present study was funded by the National Natural Science Foundation of China (grant nos. 81670504 and 81970494), the Fundamental Research Funds for the Central Universities of Central South University (grant no. 502211903) and the Key Research and Development Program of Hunan Province (grant no. 2019SK2041).

\section{Availability of data and materials}

The datasets used and/or analyzed during the present study are available from the corresponding author upon reasonable request.

\section{Authors' contributions}

XW, SP and WL performed the experiments. XM, MX, BT, $\mathrm{KN}$ and TT analyzed the data, and XW prepared the manuscript. ZS contributed to the data analysis and the revision of the manuscript. XYW contributed to the conception of the study, and guided the design and conduction of the study. All authors read and approved the final manuscript. 


\section{Ethics approval and consent to participate}

All experimental protocols were approved by the Ethics Committee of Medical Research, Third Xiangya Hospital of Central South University (Changsha, China; approval no. 2018-S092), and were performed in accordance with the National Institutes of Health Guidelines for the Care and Use of Laboratory Animals.

\section{Patient consent for publication}

Not applicable.

\section{Competing interests}

The authors declare that they have no competing interests.

\section{References}

1. Ungaro R, Mehandru S, Allen PB, Peyrin-Biroulet L and Colombel JF: Ulcerative colitis. Lancet 389: 1756-1770, 2017.

2. Ananthakrishnan AN: Epidemiology and risk factors for IBD. Nat Rev Gastroenterol Hepatol 12: 205-217, 2015.

3. Neurath MF: Current and emerging therapeutic targets for IBD. Nat Rev Gastroenterol Hepatol 14: 269-278, 2017.

4. Marchesi JR, Adams DH, Fava F, Hermes GD, Hirschfield GM, Hold G, Quraishi MN, Kinross J, Smidt H, Tuohy KM, et al: The gut microbiota and host health: A new clinical frontier. Gut 65 : 330-339, 2016

5. Sartor RB and Wu GD: Roles for intestinal bacteria, viruses, and fungi in pathogenesis of inflammatory bowel diseases and therapeutic approaches. Gastroenterology 152: 327-339.e4, 2017.

6. Shen Z, Zhu C, Quan Y, Yang J, Yuan W, Yang Z, Wu S, Luo W, Tan B and Wang X: Insights into Roseburia intestinalis which alleviates experimental colitis pathology by inducing anti-inflammatory responses. J Gastroenterol Hepatol 33: 1751-1760, 2018.

7. Tan B, Luo W, Shen Z, Xiao M, Wu S, Meng X, Wu X, Yang Z, Tian L and Wang X: Roseburia intestinalis inhibits oncostatin M and maintains tight junction integrity in a murine model of acute experimental colitis. Scand J Gastroenterol 54: 432-440, 2019.

8. Zhu C, Song K, Shen Z, Quan Y, Tan B, Luo W, Wu S, Tang K, Yang Z and Wang X: Roseburia intestinalis inhibits interleukin 17 excretion and promotes regulatory $\mathrm{T}$ cells differentiation in colitis. Mol Med Rep 17: 7567-7574, 2018

9. Kinnebrew MA, Buffie CG, Diehl GE, Zenewicz LA, Leiner I, Hohl TM, Flavell RA, Littman DR and Pamer EG: Interleukin 23 production by intestinal CD103(+)CD11b(+) dendritic cells in response to bacterial flagellin enhances mucosal innate immune defense. Immunity 36: 276-287, 2012.

10. Zhang B, Chassaing B, Shi Z, Uchiyama R, Zhang Z, Denning TL, Crawford SE, Pruijssers AJ, Iskarpatyoti JA, Estes MK, et al: Viral infection. Prevention and cure of rotavirus infection via TLR5/NLRC4-mediated production of IL-22 and IL-18. Science 346: 861-865, 2014.

11. Bergsbaken T, Fink SL and Cookson BT: Pyroptosis: Host cell death and inflammation. Nat Rev Microbiol 7: 99-109, 2009.

12. Shi J, Gao W and Shao F: Pyroptosis: Gasdermin-mediated programmed necrotic cell death. Trends Biochem Sci 42 245-254, 2017.

13. Lu A, Magupalli VG, Ruan J, Yin Q, Atianand MK, Vos MR, Schröder GF, Fitzgerald KA, Wu H and Egelman EH: Unified polymerization mechanism for the assembly of ASC-dependent inflammasomes. Cell 156: 1193-1206, 2014.

14. Wree A, Eguchi A, McGeough MD, Pena CA, Johnson CD, Canbay A, Hoffman HM and Feldstein AE: NLRP3 inflammasome activation results in hepatocyte pyroptosis, liver inflammation, and fibrosis in mice. Hepatology 59: 898-910, 2014.

15. Liu T, Zhou YT, Wang LQ, Li LY, Bao Q, Tian S, Chen MX, Chen HX, Cui J and Li CW: NOD-like receptor family, pyrin domain containing 3 (NLRP3) contributes to inflammation, pyroptosis, and mucin production in human airway epithelium on rhinovirus infection. J Allergy Clin Immunol 144: 777-787 e779, 2019.
16. Thornberry NA, Bull HG, Calaycay JR, Chapman KT, Howard AD, Kostura MJ, Miller DK, Molineaux SM, Weidner JR, Aunins J, et al: A novel heterodimeric cysteine protease is required for interleukin-1 beta processing in monocytes. Nature 356: 768-774, 1992.

17. Evavold CL, Ruan J, Tan Y, Xia S, Wu H and Kagan JC: The pore-forming protein gasdermin D regulates interleukin-1 secretion from living macrophages. Immunity 48: 35-44.e6, 2018.

18. He WT, Wan H, Hu L, Chen P, Wang X, Huang Z, Yang ZH, Zhong CQ and Han J: Gasdermin D is an executor of pyroptosis and required for interleukin-1 $\beta$ secretion. Cell Res 25: 1285-1298, 2015.

19. Davis EM, Zhang D, Glover SC, Stappenbeck T, Wang SZ and Liu JJ: Inhibition of intestinal epithelial cell pyroptosis and associated mucosal barrier defects is a potential therapeutic mechanism of action for mesalamine in Ibd. Gastroenterology 156 (Suppl): S88, 2019.

20. Ren TH,Zhou Y and Wu WY: Activation of adenosine A3 receptor inhibits Nlrp3 inflammasome and pyroptosis of colonic epithelial cells of patients with ulcerative colitis. Gastroenterology 156 (Suppl): S627, 2019.

21. Quan Y, Song K, Zhang Y, Zhu C, Shen Z, Wu S, Luo W, Tan B, Yang Z and Wang X: Roseburia intestinalis-derived flagellin is a negative regulator of intestinal inflammation. Biochem Biophys Res Commun 501: 791-799, 2018.

22. Wirtz S, Popp V, Kindermann M, Gerlach K, Weigmann B, Fichtner-Feigl S and Neurath MF: Chemically induced mouse models of acute and chronic intestinal inflammation. Nat Protoc 12: 1295-1309, 2017.

23. Livak KJ and Schmittgen TD: Analysis of relative gene expression data using real-time quantitative PCR and the 2(-Delta Delta C(T)) method. Methods 25: 402-408, 2001

24. Mangan MSJ, Olhava EJ, Roush WR, Seidel HM, Glick GD and Latz E: Targeting the NLRP3 inflammasome in inflammatory diseases. Nat Rev Drug Discov 17: 688, 2018.

25. Koelink PJ, Bloemendaal FM, Li B, Westera L, Vogels EWM, van Roest M, Gloudemans AK, van 't Wout AB, Korf H, Vermeire S, et al: Anti-TNF therapy in IBD exerts its therapeutic effect through macrophage IL-10 signalling. Gut 69: 1053-1063, 2020.

26. Na YR, Stakenborg M, Seok SH and Matteoli G: Macrophages in intestinal inflammation and resolution: A potential therapeutic target in IBD. Nat Rev Gastroenterol Hepatol 16: 531-543, 2019.

27. O'Neill LA, Sheedy FJ and McCoy CE: MicroRNAs: The fine-tuners of Toll-like receptor signalling. Nat Rev Immunol 11: 163-175, 2011.

28. Yoon SI, Kurnasov O, Natarajan V, Hong M, Gudkov AV, Osterman AL and Wilson IA: Structural basis of TLR5-flagellin recognition and signaling. Science 335: 859-864, 2012.

29. Atarashi K, Tanoue T, Oshima K, Suda W, Nagano Y, Nishikawa H, Fukuda S, Saito T, Narushima S, Hase K, et al: Treg induction by a rationally selected mixture of Clostridia strains from the human microbiota. Nature 500: 232-236, 2013.

30. Furusawa Y, Obata Y, Fukuda S, Endo TA, Nakato G, Takahashi D, Nakanishi Y, Uetake C, Kato K, Kato T, et al: Commensal microbe-derived butyrate induces the differentiation of colonic regulatory T cells. Nature 504: 446-450, 2013

31. Mardini HE and Grigorian AY: Probiotic mix VSL\#3 is effective adjunctive therapy for mild to moderately active ulcerative colitis: A meta-analysis. Inflamm Bowel Dis 20: 1562-1567, 2014.

32. Haas SL, Abbatista M, Brade J, Singer MV and Böcker U: Interleukin-18 serum levels in inflammatory bowel diseases: Correlation with disease activity and inflammatory markers. Swiss Med Wkly 139: 140-145, 2009.

33. Monteleone G, Pallone F and Caprioli F: Investigational cytokine-targeted therapies for ulcerative colitis. Expert Opin Investig Drugs 22: 1123-1132, 2013.

34. Xie Y, Xu M, Xiao Y, Liu Z, Jiang C, Kuang X, Wang C, Wu H, Peng J, Li C, et al: Treponema pallidum flagellin FlaA 2 induces IL-6 secretion in THP-1 cells via the Toll-like receptor 2 signaling pathway. Mol Immunol 81: 42-51, 2017.

35. Nakamoto $K$, Watanabe $M$, Sada $M$, Inui T, Nakamura $M$ Honda $\mathrm{K}$, Wada $\mathrm{H}$, Ishii $\mathrm{H}$ and Takizawa $\mathrm{H}$ : Pseudomonas aeruginosa-derived flagellin stimulates IL- 6 and IL-8 production in human bronchial epithelial cells: A potential mechanism for progression and exacerbation of COPD. Exp Lung Res 45: $255-266,2019$. 
36. Whitehead GS, Hussain S, Fannin R, Trempus CS, Innes CL, Schurman SH, Cook DN and Garantziotis S: TLR5 activation exacerbates airway inflammation in asthma. Lung 198: 289-298, 2020.

37. Crellin NK, Garcia RV, Hadisfar O, Allan SE, Steiner TS and Levings MK: Human CD4+ T cells express TLR5 and its ligand flagellin enhances the suppressive capacity and expression of FOXP3 in CD4+CD25+ T regulatory cells. J Immunol 175: 8051-8059, 2005.

38. Kim EH, Kim JH, Samivel R, Bae JS, Chung YJ, Chung PS, Lee SE and Mo JH: Intralymphatic treatment of flagellin-ovalbumin mixture reduced allergic inflammation in murine model of allergic rhinitis. Allergy 71: 629-639, 2016.

39. Davis EM, Kaufmann Y, Goyne H, Claggett BL, Jobin C and Liu JJ: Pyroptosis of intestinal epithelial cells Is crucial to the development of mucosal barrier dysfunction and intestinal inflammation. Gastroenterology 152 (Suppl 1): S967, 2017.

40. Kalla R, Ventham NT, Kennedy NA, Quintana JF, Nimmo ER, Buck AH and Satsangi J: MicroRNAs: New players in IBD. Gut 64: 504-517, 2015.

41. Lee S, Choi E, Cha MJ and Hwang KC: Looking for pyroptosis-modulating miRNAs as a therapeutic target for improving myocardium survival. Mediators Inflamm 2015: 254871, 2015.
42. Yang Y, Weng W, Peng J, Hong L, Yang L, Toiyama Y, Gao R, Liu M, Yin M, Pan C, et al: Fusobacterium nucleatum increases proliferation of colorectal cancer cells and tumor development in mice by activating toll-like receptor 4 signaling to nuclear Factor- $\kappa \mathrm{B}$, and up-regulating expression of MicroRNA-21. Gastroenterology 152: 851-866.e24, 2017.

43. Chassaing B, Ley RE and Gewirtz AT: Intestinal epithelial cell toll-like receptor 5 regulates the intestinal microbiota to prevent low-grade inflammation and metabolic syndrome in mice. Gastroenterology 147: 1363-1377.e17, 2014.

44. Carvalho FA, Nalbantoglu I, Aitken JD, Uchiyama R, Su Y, Doho GH, Vijay-Kumar M and Gewirtz AT: Cytosolic flagellin receptor NLRC4 protects mice against mucosal and systemic challenges. Mucosal Immunol 5: 288-298, 2012.

45. Hatai H, Lepelley A, Zeng W, Hayden MS and Ghosh S: Toll-like receptor 11 (TLR11) interacts with flagellin and profilin through disparate mechanisms. PLoS One 11: e0148987, 2016.

This work is licensed under a Creative Commons Attribution-NonCommercial-NoDerivatives 4.0 International (CC BY-NC-ND 4.0) License. 